\title{
Painless Panniculitis upon the Treatment of Clinically Amyopathic Dermatomyositis with Anti-MDA5 Antibody
}

\author{
Dai Kishida ${ }^{1}$, Satoru Ushiyama ${ }^{1}$, Yasuhiro Shimojima ${ }^{1}$, Ken-ichi Ueno ${ }^{1}$, Jun-ichi Kurashina ${ }^{1}$, \\ Takushi Shirai $^{2}$ and Yoshiki Sekijima ${ }^{1}$
}

\begin{abstract}
:
Panniculitis, a rare cutaneous manifestation in patients with dermatomyositis (DM), usually presents as a painful erythematous lesion. We herein report a 32-year-old woman with panniculitis that appeared as an indurated plaque without pain or redness after a 4-month episode of clinically amyopathic DM during treatment with prednisolone and tacrolimus. She experienced no pain; however, the firmness and extent gradually worsened. Based on our findings, including the histopathological results, DM panniculitis was diagnosed. Azathioprine was additionally administered, leading to remission. DM panniculitis can develop as a painless induration during immunosuppressive treatment, and azathioprine may be a useful treatment.
\end{abstract}

Key words: panniculitis, dermatomyositis, azathioprine, anti-MDA5 antibody

(Intern Med 60: 2697-2700, 2021)

(DOI: 10.2169/internalmedicine.6931-20)

\section{Introduction}

Dermatomyositis (DM) is a chronic inflammatory disorder characterized by various cutaneous lesions, along with muscle and lung involvement. Panniculitis is a rare manifestation in patients with DM and reportedly presents as a painful erythematous nodule or plaque $(1,2)$. However, panniculitis without remarkable pain or redness is not well known.

We herein report a patient with panniculitis that appeared as an indurated plaque without pain or redness during clinically amyopathic DM (CADM) treatment, which was successfully treated with methotrexate and azathioprine.

\section{Case Report}

A 32-year-old woman was referred to our hospital because of a skin lesion and joint pain. A physical examination revealed periungual erythema, mechanic's hands, mild alopecia, facial erythema, and painful erythema on her right thigh (Fig. 1a, b). There was no muscle weakness or myalgia, and no rales were heard.

Laboratory examinations revealed slight elevations in her levels of creatine kinase (169 U/L; normal, 30-165 U/L), aldolase (8.2 U/L; normal, 2.5-7.5 U/L), serum ferritin (153.0 $\mathrm{ng} / \mathrm{mL}$; normal, $10-120 \mathrm{ng} / \mathrm{mL})$, C-reactive protein $(0.32$ $\mathrm{mg} / \mathrm{dL}$; normal, $<0.10 \mathrm{mg} / \mathrm{dL}$ ), and lactate dehydrogenase (327 U/L; normal, 120-230 U/L). The Krebs von den Lungen-6 (369 U/mL; normal, 105-435 U/mL) levels were not elevated. However, a high titer of anti-MDA5 antibody (147.0 index; normal, <32) was observed. Anti-nuclear antibody and other autoantibodies, except for the anti-MDA5 antibody, were negative. Chest computed tomography indicated slight peripheral consolidations (Fig. 1c). A skin biopsy of the right thigh revealed parakeratosis, liquefactive degeneration below the epidermis, and mild lymphoid infiltration in the perivascular area of the dermis, which was consistent with DM (Fig. 1d). Mucin deposition was not observed, and there was no remarkable change in the fat tissue (Fig. 1e). Given these findings, we established a diagnosis of CADM according to the definition of DM-related terms (3). The patient was treated with prednisolone $(60 \mathrm{mg} /$ day) and tacrolimus ( $3 \mathrm{mg} /$ day), and gradual improvement was observed in the cutaneous lesions and joint pain. Prednisolone was tapered, and she was discharged after one month.

\footnotetext{
${ }^{1}$ Department of Medicine (Neurology \& Rheumatology), Shinshu University School of Medicine, Japan and ${ }^{2}$ Department of Dermatology, Shinshu University School of Medicine, Japan

Received: December 14, 2020; Accepted: January 25, 2021; Advance Publication by J-STAGE: March 8, 2021

Correspondence to Dr. Dai Kishida, dkishida@shinshu-u.ac.jp
} 

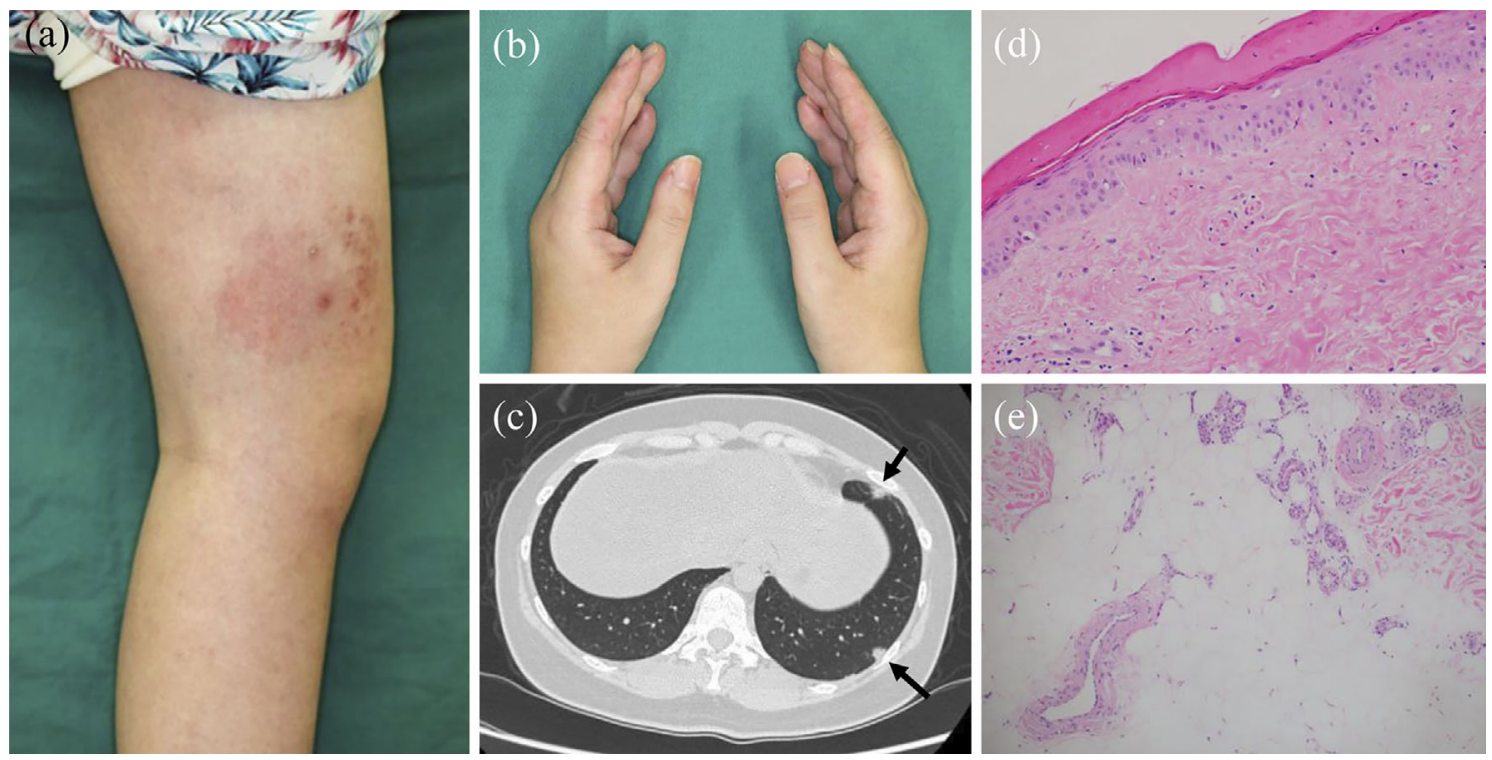

Figure 1. Cutaneous and lung lesions at the first visit. (a) Painful erythema on the right thigh. (b) Periungual erythema and mechanic's hands. (c) Peripheral consolidations on chest computed tomography (arrows). (d, e) Histopathology of the first biopsy from an erythema on the thigh. (d) Parakeratosis, liquefactive degeneration below the epidermis, and mild lymphoid infiltration in the perivascular area of the dermis [Hematoxylin and Eosin $(H \& E)$ staining, $\times 200]$. (e) No remarkable changes in the fat tissue (H\&E staining, $\times 100)$.
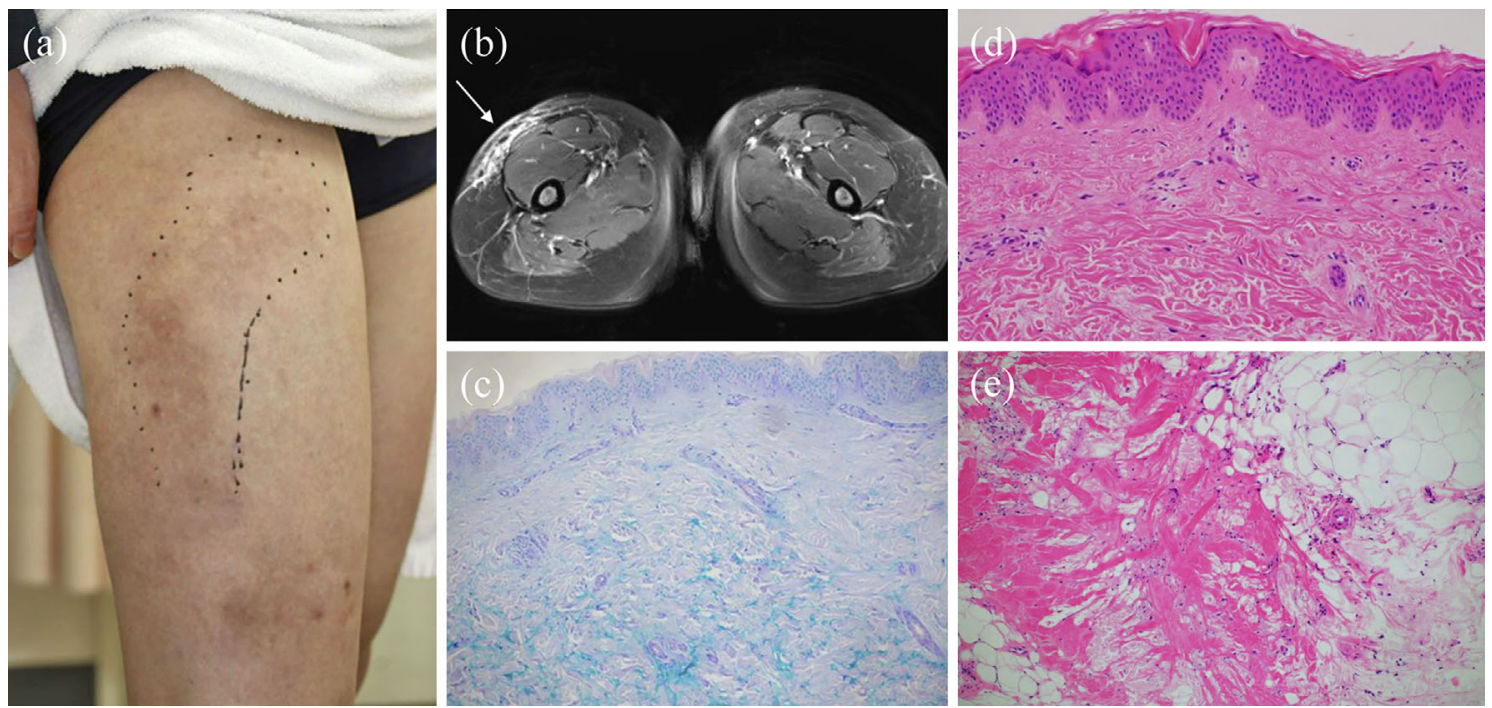

Figure 2. (a) An indurated plaque without pain on the lateral side of the thigh, slightly proximal to the initial lesion (marker). (b) A high-intensity area noted subcutaneously on fat-suppressed T2weighted magnetic resonance imaging (arrow). (c-e) Histopathology of the second biopsy from an induration. (c) Interstitial mucin deposition between collagen bundles in the dermis (Alcian Blue-PAS stain, $\times 100$ ). (d) Thickening of the epidermis and fibrous hyperplasia in the dermis [Hematoxylin and Eosin $(H \& E)$ staining, $\times 200]$. (e) Fibrosis and mild lymphoid infiltration in the fat tissue that was not observed at first biopsy $(\mathrm{H} \& \mathrm{E}$ staining, $\times 100)$.

Three months after discharge, however, while still taking $16 \mathrm{mg}$ of prednisolone and $4 \mathrm{mg}$ of tacrolimus daily, the patient noticed an induration on the lateral side of her right thigh, slightly proximal to the initial erythema (Fig. 2a). She did not experience any pain, except when it was strongly compressed. Another small induration also appeared on the left thigh and buttocks, and the firmness and extent of the lesions gradually worsened. Magnetic resonance imaging showed a high-intensity area subcutaneously on fatsuppressed T2-weighted images, which was faintly contrasted (Fig. 2b). A skin biopsy revealed thickening of the epidermis as well as mucin deposition and collagen fiber hy- 


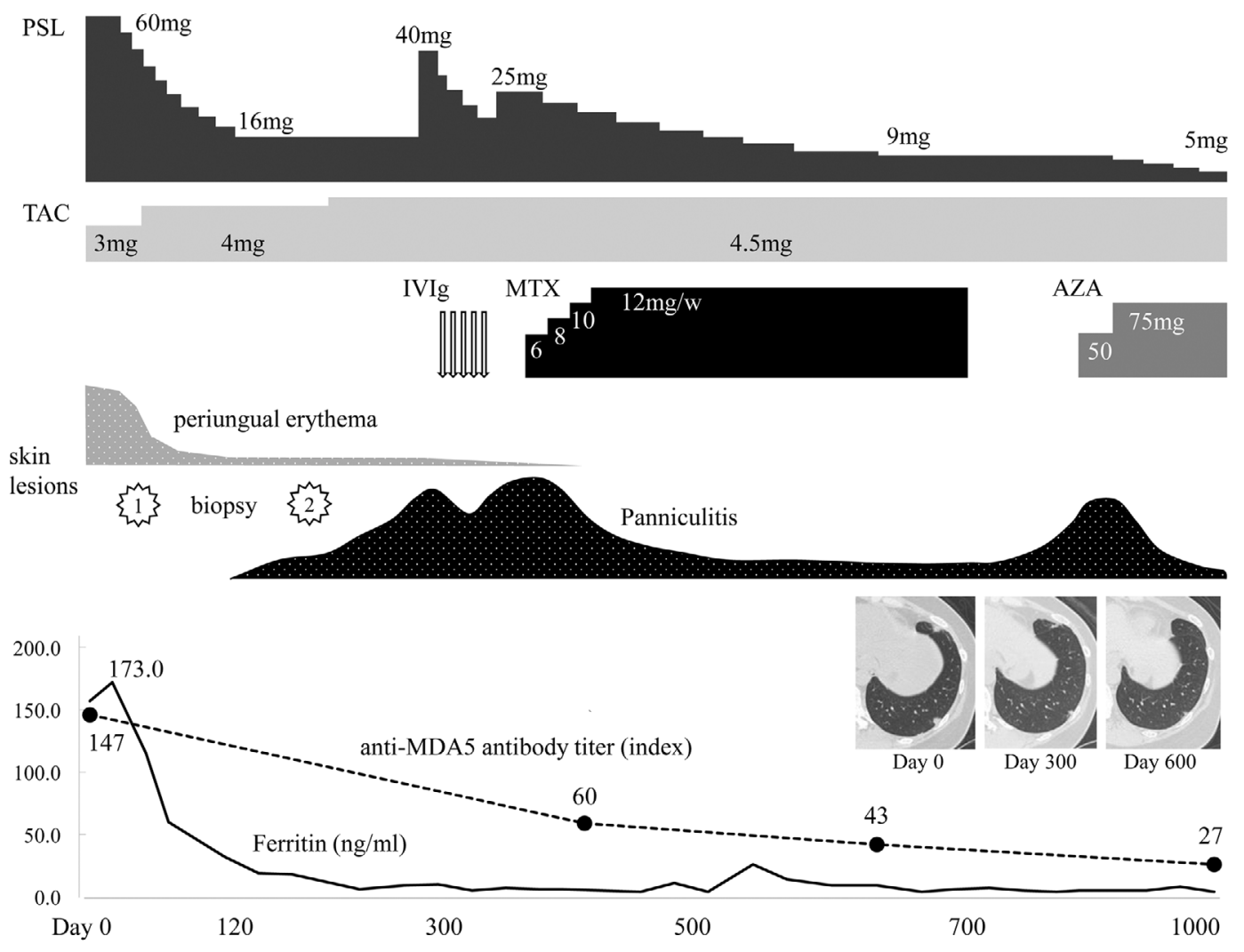

Figure 3. Clinical course of the patient. PSL: prednisolone, TAC: tacrolimus, IVIg: intravenous immunoglobulin, MTX: methotrexate, AZA: azathioprine

perplasia of the dermis (Fig. 2c, d). Furthermore, fibrosis with mild lymphoid infiltration was observed mostly at the septa of the fat tissue (Fig. 2e). Based on these findings, we established a diagnosis of panniculitis associated with DM. Other cutaneous manifestations, such as periungual erythema and mechanic's hands, were not exacerbated. The patient was treated with an increased dose of prednisolone (40 mg/ day) and intravenous immunoglobulin. There was a temporary improvement in the subcutaneous indurations; however, the area and firmness of the lesions worsened when prednisolone was reduced to $\leq 20 \mathrm{mg} /$ day. Although the additional use of methotrexate was effective, the lesions worsened again when methotrexate was discontinued because of the patient's wish to become pregnant. We added azathioprine, which resulted in evident improvement in the indurations, and reduced the dosage of prednisolone (Fig. 3). The lesions continued to be mildly firm; however, prednisolone was reduced to $5 \mathrm{mg} /$ day with no worsening of the lesions. Peripheral consolidations of the lung were gradually improved throughout the course of treatment.

\section{Discussion}

We herein report a patient with panniculitis associated with CADM that developed as an indurated plaque without pain during treatment with prednisolone and tacrolimus. Panniculitis is reported to occur as painful nodules on the buttocks, arms, abdomen, and thighs and rarely manifests in patients with DM (1). Panniculitis can develop at any time during DM, either as an initial symptom or during treatment (4). The histopathologic findings are reportedly similar to those of lupus panniculitis, lobular panniculitis with lymphocytic infiltrate, mucin deposition between collagen bundles, and sclerotic changes in the septa $(5,6)$. In the present case, panniculitis occurred during treatment near the initial erythema but was quite different clinically and histopathologically. It developed silently, without pain or infiltration of lymphocytic cells. The pathogenesis of panniculitis in DM remains unclear; however, the features of our patient may reflect the condition of receiving immunosuppressive treatment with moderate doses of prednisolone and tacrolimus.

Anti-MDA5 antibody is a specific myositis-associated autoantibody, reported to be associated with a high frequency of rapidly progressive interstitial lung disease and joint involvement (7). Regarding cutaneous findings, palmar papules, ulceration, lateral digit hyperkeratosis, and alopecia have been reported as distinctive lesions (8). The titer of anti-MDA5 antibody is reported to correlate with the disease activity in patients with DM (9), and in this case, the titer decreased with improvement in panniculitis and lung lesions. Some reports have discussed the association between panniculitis and MDA5 $(10,11)$; however, the number of patients has been small, and further studies are needed. We found five case reports of patients with anti-MDA5 antibody-positive DM complicated with panniculitis, and all 
patients had panniculitis as their initial symptom (12-16). Our case showed that patients with anti-MDA5 antibodypositive DM can develop panniculitis even during treatment.

At present, there is no established treatment for panniculitis in DM. High-dose corticosteroids are the main treatment in most cases, and various drugs are used empirically, such as methotrexate (17), hydroxychloroquine (18), and intravenous immunoglobulin $(19,20)$. Our patient developed panniculitis under treatment with prednisolone and tacrolimus. The effectiveness of increasing the dose of prednisolone and intravenous immunoglobulin was temporary, and methotrexate had to be discontinued despite showing a good response because the patient wished to get pregnant. There are limited reports on the effectiveness of azathioprine in DM panniculitis (21); however, azathioprine is reportedly useful, both initially and during maintenance therapy for DM, and contributes to survival benefits. Furthermore, the recommendation of the European League against Rheumatism classified azathioprine as a drug that could be continued during pregnancy and lactation (22). This case showed that azathioprine is a useful option in the treatment of DM panniculitis, especially for women of child-bearing age.

In conclusion, we encountered a patient with DM panniculitis that developed as an indurated plaque without pain during immunosuppressive treatment. The effectiveness of prednisolone, tacrolimus, and intravenous immunoglobulin was insufficient; however, azathioprine showed a good effect of improving the firmness and extent of the lesion. Panniculitis should be suspected when an indurated plaque is observed in patients with DM, even if it is not painful. Similarly, azathioprine may be useful in the treatment of DM panniculitis, including for women of child-bearing age.

The authors state that they have no Conflict of Interest (COI).

\section{References}

1. Mainetti C, Beretta-Piccoli BT, Selmi C. Cutaneous Manifestations of Dermatomyositis: a Comprehensive Review. Clin Rev Allergy Immunol 53: 337-356, 2017.

2. Hansen CB, Callen JP. Connective tissue panniculitis: lupus panniculitis, dermatomyositis, morphea/scleroderma. Dermatol Ther 23: 341-349, 2010.

3. Gerami P, Schope JM, McDonald L, et al. A systematic review of adult-onset clinically amyopathic dermatomyositis (dermatomyositis sine myositis): a missing link within the spectrum of the idiopathic inflammatory myopathies. J Am Acad Dermatol 54: 597613, 2006.

4. Braunstein I, Werth VP. Update on management of connective tissue panniculitides. Dermatol Ther 25: 173-182, 2012.

5. Requena L, Sanchez Yus E. Panniculitis. Part II. Mostly lobular panniculitis. J Am Acad Dermatol 45: 325-361, 2001.

6. Santos-Briz A, Calle A, Linos K, et al. Dermatomyositis pannicu- litis: a clinicopathological and immunohistochemical study of 18 cases. J Eur Acad Dermatol Venereol 32: 1352-1359, 2018.

7. Moghadam-Kia S, Oddis CV, Aggarwal R. Anti-MDA5 antibody spectrum in western world. Curr Rheumatol Rep 20: 78, 2018.

8. Kurtzman DJB, Vleugels RA. Anti-melanoma differentiationassociated gene 5 (MDA5) dermatomyositis: a concise review with an emphasis on distinctive clinical features. J Am Acad Dermatol 78: 776-785, 2018.

9. Sato S, Kuwana M, Fujita T, Suzuki Y. Anti-CADM-140/MDA5 autoantibody titer correlates with disease activity and predicts disease outcome in patients with dermatomyositis and rapidly progressive interstitial lung disease. Mod Rheumatol 23: 496-502, 2013.

10. Fiorentino D, Chung L, Zwerner J, Rosen A, Casciola-Rosen L. The mucocutaneous and systemic phenotype of dermatomyositis patients with antibodies to MDA5 (CADM-140): a retrospective study. J Am Acad Dermatol 65: 25-34, 2011.

11. Labrador-Horrillo M, Martinez MA, Selva-O'Callaghan A, et al. Anti-MDA5 antibodies in a large Mediterranean population of adults with dermatomyositis. J Immunol Res 2014: 290797, 2014.

12. Hasegawa A, Shimomura Y, Kibune N, Koshio J, Umemori Y, Abe R. Panniculitis as the initial manifestation of dermatomyositis with anti-MDA5 antibody. Clin Exp Dermatol 42: 551-553, 2017.

13. Galli S, Naudi G, Guler SA, et al. Kissed by MDA-5: lobular panniculitis of the cheek as an initial symptom of dermatomyositis. Rheumatology 59: 1189, 2019.

14. Hattori Y, Matsuyama K, Takahashi T, Shu E, Kanoh H, Seishima M. Anti-MDA5 antibody-positive dermatomyositis presenting with cellulitis-like erythema on the mandible as an initial symptom. Case Rep Dermatol 10: 110-114, 2018.

15. Agullo A, Hinds B, Larrea M, Yanguas I. Livedo Racemosa, reticulated ulcerations, panniculitis and violaceous plaques in a 46year-old woman. Indian Dermatol Online J 9: 47-49, 2018.

16. Pau-Charles I, Moreno PJ, Ortiz-Ibáñez K, et al. Anti-MDA5 positive clinically amyopathic dermatomyositis presenting with severe cardiomyopathy. J Eur Acad Dermatol Venereol 28: 1097-1102, 2014.

17. Azevedo PO, Castellen NR, Salai AF, Barbosa FS, Alves C, Pinto CAL. Panniculitis associated with amyopathic dermatomyositis. An Bras Dermatol 93: 119-121, 2018.

18. Abdul-Wahab A, Holden CA, Harland C, Patel S. Calcific panniculitis in adult-onset dermatomyositis. Clin Exp Dermatol 34: e854-856, 2009.

19. Sabroe RA, Wallington TB, Kennedy CT. Dermatomyositis treated with high-dose intravenous immunoglobulins and associated with panniculitis. Clin Exp Dermatol 20: 164-167, 1995.

20. Carroll M, Mellick N, Wagner G. Dermatomyositis panniculitis: a case report. Australas J Dermatol 56: 224-226, 2015.

21. Carrera E, Lobrinus JA, Spertini O, Gherardi RK, Kuntzer T. Dermatomyositis, lobar panniculitis and inflammatory myopathy with abundant macrophages. Neuromuscul Disord 16: 468-471, 2006.

22. Skorpen CG, Hoeltzenbein M, Tincani A, et al. The EULAR points to consider for use of antirheumatic drugs before pregnancy, and during pregnancy and lactation. Ann Rheum Dis $\mathbf{7 5}$ : 795-810, 2016.

The Internal Medicine is an Open Access journal distributed under the Creative Commons Attribution-NonCommercial-NoDerivatives 4.0 International License. To view the details of this license, please visit (https://creativecommons.org/licenses/ by-nc-nd/4.0/).

(C) 2021 The Japanese Society of Internal Medicine

Intern Med 60: 2697-2700, 2021 